\title{
A spin network generalization of the Jones Polynomial and Vassiliev invariants
}

\author{
Rodolfo Gambini ${ }^{1 *}$, Jorge Griego ${ }^{1}$, Jorge Pullin ${ }^{2}$ \\ 1. Instituto de Física, Facultad de Ciencias, Tristan Narvaja 1674, Montevideo, Uruguay \\ 2. Center for Gravitational Physics and Geometry, Department of Physics, \\ The Pennsylvania State University, 104 Davey Lab, University Park, PA 16802
}

(Nov 8th, 1997)

\begin{abstract}
We apply the ideas of Alvarez and Labastida to the invariant of spin networks defined by Witten and Martin based on Chern-Simons theory. We show that it is possible to define ambient invariants of spin networks that (for the case of $S U(2)$ ) can be considered as extensions to spin networks of the Jones polynomial. Expansions of the coefficients of the polynomial yield primitive Vassiliev invariants. The resulting invariants are candidates for solutions of the Wheeler-DeWitt equations in the spin network representation of quantum gravity.
\end{abstract}

Witten [1] showed that the expectation value of a Wilson loop in an $S U(2)$ Chern-Simons theory,

$$
<W(\gamma)>=\int D A W(\gamma, A) \exp \left(\frac{i k}{4 \pi} \int d^{3} x \operatorname{Tr}\left(A \wedge \partial A+\frac{2}{3} A \wedge A \wedge A\right)\right)
$$

is a knot invariant closely related with the Jones polynomial. More precisely, it is given by the Kauffman bracket [2] knot polynomial. The latter differs from the Jones polynomial by an overall "phase factor" given by the exponential of the writhe of the knot. This factor accounts for the fact that the Kauffman bracket is a regular isotopy invariant (it is not invariant when one removes twists, i.e., it is an invariant of framed knots) whereas the Jones polynomial is an ambient isotopic invariant. The explicit relation between the Kauffman bracket and the expectation value we introduced above is given by $K(\gamma, q)=<W(\gamma)>$ with $q=\exp (2 \pi i / k)$, where $q$ is the variable of the polynomial and $k$ is the coupling constant of the Chern-Simons theory ${ }^{\mathrm{t}}$.

The expression of the invariants one obtains from the path integral appear as power series expansions in terms of the parameter $2 \pi i / k$. It is tantamount to evaluating the polynomials for $q=\exp (2 \pi i / k)$ and expanding the exponential in power series. If one does this for the Jones polynomial, the resulting coefficients are Vassiliev invariants [5 7].

In a separate context, it was also noticed that the resulting Vassiliev invariants are candidates for wavefunctions in the loop representation of quantum gravity 8 11]. In this context, however, one is not interested in arbitrary knot invariants, but actually in those that arise as counterparts of states in the "connection representation",

$$
\psi(\gamma)=\int D A W(\gamma, A) \psi(A)
$$

This poses a series of restrictions on the possible knot invariants $\psi(\gamma)$ that one could consider as wavefunctions of a quantum theory in the loop representation. These restrictions are known as "Mandelstam identities". If one concentrates of wavefunctions depending on a single loop, they are linear, loop dependent, relations $\sum_{i} c_{i} \psi\left(\gamma_{i}\right)=0$. They arise from the fact that the Wilson loop $W(\gamma, A)$ is a trace of a group element [12]. Of all the Vassiliev invariants that one constructs using the techniques described above, we only know for a fact that two of them are compatible with these relations. This makes it particularly difficult to construct candidates for quantum states of the gravitational field in terms of these invariants. Implicit in this discussion is the fact that in quantum gravity one is interested in loops with generic intersections, otherwise the spectra of various quantum operators is not appropriately realized [13]. Most of the discussions of knot invariants stemming from Chern-Simons theories have concentrated on smooth non-intersecting loops or multiloops.

Given the limitations imposed by the Mandelstam identities and their complicated nature, it has proven very fruitful to consider holonomies in all possible representations of the group. In a sense this is tantamount to dealing with "all possible loops, multiloops and intersections" in a unified framework [14]. This leads to the construction of generalized gauge invariant quantities dependent on trivalent (or higher) graphs [15, 14, 16]. These invariants can

\footnotetext{
*Associate member of ICTP.

${ }^{1}$ There has been some controversy [3] concerning the relationship of $q$ and $k$. Nonperturbative effects seem to fix the value to

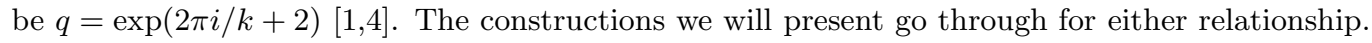


be thought of as "Wilson nets" (generalizations of the Wilson loops dependent on a spin network), and one can therefore consider computing their expectation value in a Chern-Simons theory. This was first attempted by Witten [15] and further developed by Martin [17]. These invariants are related to families of invariants constructed using braid group techniques by Kauffman and Lins [18]. In a recent paper we have considered related invariants as possible candidates for states of the quantum gravitational field [16]. Unfortunately, a significant limitation of these invariants is that they are regular isotopic invariants, that is, they are framing-dependent. Therefore they cannot be genuinely considered quantum states of the gravitational field, since the latter are supposed to be diffeomorphism invariant, that is, unchanged by any smooth deformation of the loops (including twists).

The purpose of this note is to address the latter issue. We will make use of a series of observations by Alvarez and Labastida 19] that will allow us to construct an ambient isotopic invariant from the expectation value of a Wilson-net. The resulting invariant will be related to the Jones polynomial and will allow us to introduce Vassiliev invariants in the context of spin nets. These invariants are therefore candidates for wavefunctions of the quantum gravitational field.

Returning to the single-loop (ie, not spin-net) context, it was noted by Alvarez and Labastida [19], that one could re-express the expectation value of the Wilson loop as,

$$
<W(\gamma)>=K_{0}(\gamma) \exp \left(\sum_{i=1}^{\infty} \sum_{j=1}^{d_{i}} \alpha_{i j}(\gamma) r_{i j}\left[\frac{2 \pi i}{k}\right]^{i}\right),
$$

where $r_{i j}$ are group-dependent constants (the construction goes through for any group) and $\alpha_{i j}(\gamma)$ are the "primitive" Vassiliev invariants ${ }^{2}$. These are the minimal set of invariants for a given order that are needed to generate all invariants of higher orders via products. What has been accomplished here is a re-summation of the power series that arises in computing the expectation value, into an exponential involving a linear combination of the primitive Vassiliev invariants. This resummation can be straightforwardly understood in terms of Feynman diagrammatics, through the usual procedure to generate connected diagrams by writing the generating function as an exponential [20].

Based on this resummation, it is immediate to define an ambient isotopic invariant for multiloops $P(\gamma, k)$, simply by removing the framing-dependent factor explicitly, i.e.,

$$
\begin{aligned}
<W(\gamma)> & =\exp \left(\alpha_{11} r_{11}\left[\frac{2 \pi i}{k}\right]\right) K_{0}(\gamma) P(\gamma, k) \\
P(\gamma, k) & =\exp \left(\sum_{i=2}^{\infty} v_{i}(\gamma)\left[\frac{2 \pi i}{k}\right]^{i}\right) \\
v_{i} & =\sum_{j=1}^{d_{i}} \alpha_{i j}(\gamma) r_{i j} .
\end{aligned}
$$

This expression very clearly embodies the different properties of transformation of the invariant. The exponential prefactor is responsible for the framing dependence (non-invariance under twists). The $K_{0}(\gamma)$ factor takes care of the fact that the invariant changes by a power of $(-1)$ when one "flips over" an entire knot through a diffeomorphism, since the orientation of all trivalent intersections is reversed (see below).

What we will do in this paper is to follow a similar procedure in the context of spin networks for the $S U(2)$ group. What allows us to do this is that the formulae (4) have been shown to hold for multi-loops [19]. We will assume they hold in the case of intersecting loops, and therefore for spin-nets. This will allow us to define an ambient isotopic invariant associated with Chern-Simons theory. In order to do this, we start by recalling the invariant constructed by Witten and Martin [15,17]. It is defined by the following skein relations,

$$
\Delta_{j}=E(j, k)=\frac{q^{j+\frac{1}{2}}-q^{-j-\frac{1}{2}}}{q^{\frac{1}{2}}-q^{-\frac{1}{2}}}
$$

\footnotetext{
${ }^{2}$ With the addition of $\alpha_{11}$, which is the framing-dependent factor, which would be the only contribution to the expectation value if one considered an Abelian theory.
} 


$$
\begin{aligned}
& \Theta\left(j_{1}, j_{2}, j\right)=E\left(j\left(\begin{array}{ll}
j_{1} \\
j_{2}
\end{array}\right), k\right)=\sqrt{\Delta_{1} \Delta_{2} \Delta_{3}} \\
& E\left(Y_{j_{3}}^{j_{1}} j_{2}^{j_{2}}\right)=(-1)^{j_{1}+j_{2}+j_{3}} \exp \left(i \pi\left(h_{1}+h_{2}-h_{3}\right)\right) E\left(\bar{j}_{1} \bigodot_{j_{3}} j_{2, k}\right), \quad h_{i} \equiv \frac{j_{i}\left(j_{i}+1\right)}{k} \\
& E(j \uparrow, k)=\exp \left(-2 \pi i h_{j}\right) E(j \uparrow \bigcirc, k)=\exp \left(2 \pi i h_{j}\right) E(j \uparrow, k)
\end{aligned}
$$

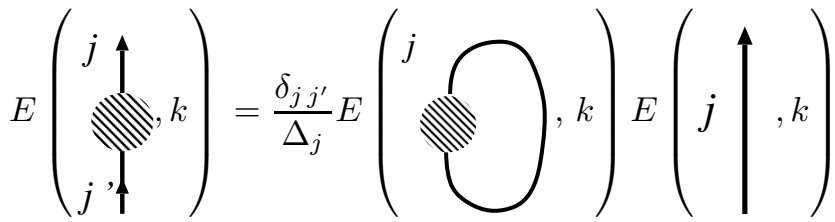

$$
\begin{aligned}
& \left.E\left(j_{j_{2}} j_{j_{3}}^{j_{1}}, k\right)=\frac{1}{\sqrt{\Delta_{1} \Delta_{2} \Delta_{3}}} E(\sum_{j_{2}} \overbrace{j_{2}}^{j_{j_{3}}}), k\right) \\
& E\left(\begin{array}{l}
j_{1} \\
j_{2}
\end{array}\right)=\sum_{i=\left|j_{1}-j_{2}\right|}^{i=\left|j_{1}+j_{2}\right|} \sqrt{\frac{\Delta_{i}}{\Delta_{1} \Delta_{2}}} E(\underbrace{j_{i}}_{j_{1}}, k) \\
& E\left(j_{1} j_{j_{2}}, k\right)=\sum_{i=\left|j_{1}-j_{2}\right|}^{i=\left|j_{1}+j_{2}\right|} \sqrt{\frac{\Delta_{i}}{\Delta_{1} \Delta_{2}}}(-1)^{j_{1}+j_{2}+j_{3}} \exp \left(i \pi\left(h_{1}+h_{2}+h_{3}\right)\right) E(\underbrace{\left.\sum_{j_{i}}^{j_{1}}, k\right)}_{j_{1}} \hat{j}^{j_{2}}) \\
& E\left(\begin{array}{l}
j_{j} \\
j_{2} \\
j_{3}
\end{array}\right)=\sum_{j=\left|j_{1}-j_{4}\right|}^{j_{4}}\left\{\begin{array}{lll}
j_{2} & j_{1} & j \\
j_{4} & j_{3} & l
\end{array}\right\}_{q} E(\underbrace{j_{1}+j_{4} \mid}_{j_{2}} \bigwedge_{j_{3}}^{j_{l}}, k),
\end{aligned}
$$

where the expression in curly braces is the q-deformed Racah symbol, which is defined as,

$$
\left\{\begin{array}{lll}
j_{2} & j_{1} & j \\
j_{4} & j_{3} & l
\end{array}\right\}_{q}=\frac{E\left(j_{j} j_{j}, k\right.}{j_{\Delta_{1} \Delta_{2} \Delta_{3} \Delta_{4}}} .
$$

The above expressions completely characterize the invariant for any spin network. The explicit expression for the value of the invariant in the last expression "the tetrahedron diagram" can be computed using the recoupling identity (15) in conjunction with the definitions of the Delta and Theta diagrams (77,8) (see [17] for an explicit computation). 
This invariant is closely related to that of Kauffman and Lins [18], but has two main differences. The first one is the choice in the definition of the "Wilson net" gauge invariant object. These objects are defined by joining holonomies along the lines of the net at the vertices using group invariant tensors. There is an ambiguity in the definition, given by which line ends up being contracted into which entry of the $3 \mathrm{jm}$ coefficients (closely related to the Clebsch-Gordan coefficients) [21]. This ambiguity can be coded into an "orientation" of the lines entering the intersection, due to the cyclic property of the coefficients. If one performs a diffeomorphism on a diagram, one in general can reverse the orientation of an intersection. Witten and Martin [15,17] introduce an additional normalization factor in the definition of the Wilson net in such a way as to make them invariant under changes of orientation at the vertices. The coefficient is,

$$
V_{ \pm}=\exp \left( \pm \frac{i \pi}{2}\left[j_{1}+j_{2}+j_{3}\right]\right) \sqrt[4]{\left(2 j_{1}+1\right)\left(2 j_{2}+1\right)\left(2 j_{3}+1\right)}
$$

where the \pm refers to the two possible orientations of a trivalent vertex. Kauffman and Lins do not introduce this normalization factor. The invariant they construct is therefore less naturally associated with an expectation value of a Wilson net (their construction is entirely in terms of braid group techniques, and does not concern itself with viewing the invariant as an expectation value of a Wilson net). From our point of view, since we are interested in constructing states of quantum gravity using these invariants, it is therefore natural to choose the normalization of Witten and Martin.

The second difference between the Witten-Martin and the Kauffman-Lins invariants is given by the definition of the planar diagrams (7,8). This difference can be understood [15] in terms of the freedom that one has in choosing the measure to define the path integral, and the two definitions can be viewed as a choice of measure. It is not clear at the moment which is the "correct" measure for quantum gravity. Although there is progress in understanding diffeomorphism invariant measures [22], these have proved ineffective at the time of performing integrals like the expectation value of the Wilson net in a Chern-Simons theory. Variational techniques [23].16], which only involve weak assumptions about the measure considered for evaluating the path integral, yield unique results for the identities involving crossings and twists, but up to now they have failed to produce unique results for the recoupling identities and the evaluation of the planar diagrams.

We now proceed to apply the technique of Alvarez and Labastida to the invariant we just introduced. The first step is to understand the moves that are associated with diffeomorphisms, since one would expect the final object to be invariant under diffeomorphisms. The moves in equations (9) and (10) are associated with diffeomorphisms. In addition to these, one has to be concerned with twists at intersections, similar to those in equation (9) but involving several strands, specifically,

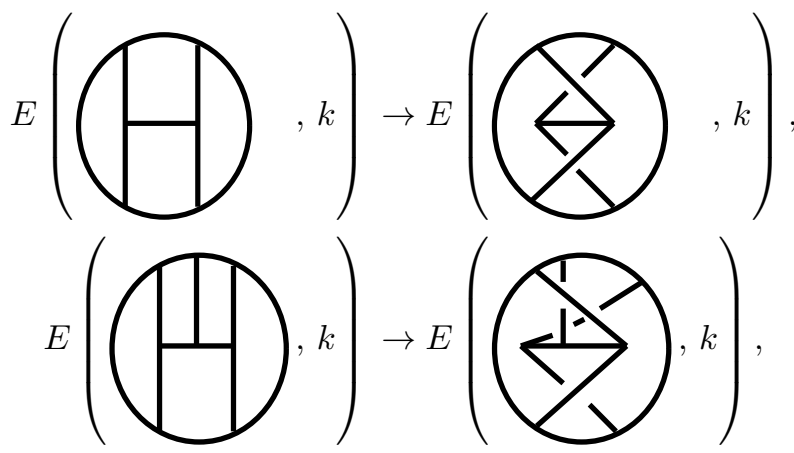

and similarly for $n$ insertions.

The first task consists in identifying the equivalent of $v_{1} \equiv r_{11} \alpha_{11}$ in equation (雨), which we can easily do by considering the expansion of $E(\Gamma, k)$ to first order in $\kappa=\frac{2 \pi i}{k}$

$$
E(\Gamma, \kappa)=E(\Gamma, 0)+\kappa E(\Gamma, 0) v_{1}(\Gamma)+\cdots
$$

If one now considers equation (10) and its expansion to first order, it is immediate to see that,

$$
v_{1}(j \uparrow)=-j(j+1)+v_{1}(j \uparrow \bigcirc)=j(j+1)+v_{1}(j \bigcirc)
$$


What we now see is that if one defines an invariant $P$ as we introduced in equation (4), the skein relations we just discussed for $v_{1}$ compensate exactly the changes that the invariant $E$ underwent under twists as in equation (10). It is straightforward to see that this is also true for twists at the intersections as those shown in equation (9). The only task left is to show that this is also true for twists with many insertions of lines, as those shown in (18.,19) and their generalization for $n$ lines. The way to show that these twists can be "undone" via the application of the fundamental Reidemeister moves and the invariance under the twist at a trivalent vertex we have just discussed. Specifically, let us consider the following move,

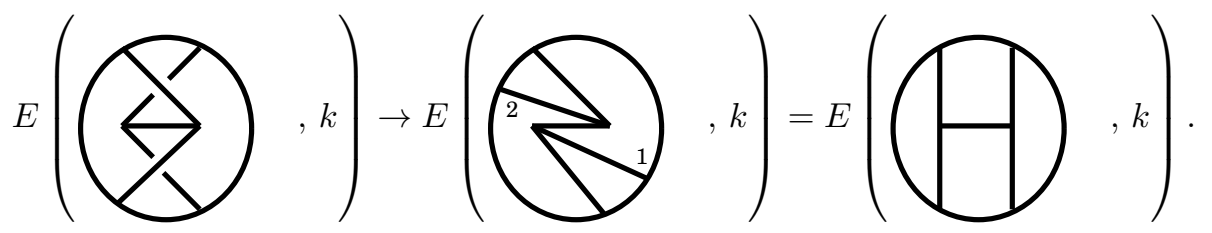

What occurred here is that line number 1 was "slid" under the rest of the lines towards the bottom. This is a valid Reidemeister move as long as the orientation of the trivalent intersection of the left is kept unchanged. Once the line number 1 slides below the horizontal line, one is introducing a twist of the form (10). We have just shown that the invariant does not change under such twists. From there on one keeps on performing a Reidemeister move. A similar discussion applies to the line number 2 . We end up with a diagram topologically equivalent to the untwisted one. This discussion can be easily generalized to the case of twists involving $n$ inserted lines.

We have therefore constructed an invariant polynomial which admits an expansion in terms of the analog for spin-networks of Vassiliev invariants. Functions of these invariants are natural candidates for states of the quantum gravitational field in the loop representation of quantum gravity. In particular, they are diffeomorphism invariant, compatible with the Mandelstam identities of $S U(2)$ and particularize for the case of single loops to invariants that in some particular cases have been found to be annihilated by the Hamiltonian constraint of quantum gravity.

It has been conjectured [5] that Vassiliev invariants could be able to separate all knots. The basis of this conjecture is that it is true for braids. This suggests that any knot invariant could be expanded in terms of these invariants. If one believes this conjecture, one should conclude that these invariants would constitute an ideal setting for discussing quantum gravity in the loop representation. An important issue to be addressed, as pointed out by Kauffman [24] is if all Vassiliev invariants arise from Chern-Simons theory. If this were the case, then the actual setting for quantum gravity could be given completely by the invariants we have been discussing. It is now known that all Vassiliev invariants can be obtained from Chern-Simons theory [25]. In order to discuss the invariants of interest in quantum gravity one has to specify a given gauge group, $S U(2)$. It is still questionably if the invariants that are obtained from a single gauge group are "general enough" to span the whole space of states of quantum gravity. This issue is currently under consideration.

Summarizing, we have constructed a family of ambient isotopic knot invariants that are candidates for quantum states of the gravitational field in the spin network representation. It is yet to be established if this family is a complete enough "arena" for the quantization of general relativity.

We wish to thank Viqar Husain and José Labastida for pointing us to the work of Witten and Martin. We are also grateful to Laurent Freidel for discussions. This work was supported in part by grants NSF-INT-9406269, NSFPHY-9423950, research funds of the Pennsylvania State University, the Eberly Family research fund at PSU and PSU's Office for Minority Faculty development. JP acknowledges support of the Alfred P. Sloan foundation through a fellowship. We acknowledge support of Conicyt (project 49) and PEDECIBA (Uruguay).

[1] E. Witten, Commun. Math. Phys 121, 351 (1989).

[2] L. Kauffman "Knots and physics", World Scientific Series on Knots and Everything 1, World Scientific, Singapore (1991).

[3] E. Guadagnini, "The link invariants of the Chern-Simons field theory, new developments in topological quantum field theory", De Gruyter expositions in mathematics, 10, W. De Gruyter, New York (1993).

[4] M. Awada, Comm. Math. Phys. 129, 329 (1990).

[5] D. Bar-Natan, Topology, 34, 423 (1995); q-alg/9702009.

[6] J. Birman, X. Lin, Inv. Math. 111, 225 (1993).

[7] J. Baez, Lett. Math. Phys. 26, 43 (1992).

[8] B. Brügmann, R. Gambini, J. Pullin, Phys. Rev. Lett. 68, 431 (1992). 
[9] B. Brügmann, R. Gambini, J. Pullin, Nucl. Phys. B385, 587 (1992).

[10] B. Brügmann, R. Gambini, J. Pullin, Gen. Rel. Grav. 25, 1 (1993).

[11] Gambini, R., Pullin, J.: "Loops, knots, gauge theories and quantum gravity." Cambridge: Cambridge University Press, Cambridge (1996).

[12] R. Gambini, A. Trias, Nucl. Phys. B278, 436 (1986).

[13] Brügmann, B., Pullin, J.: Nucl. Phys. B363, 221 (1991).

[14] C. Rovelli, L. Smolin, Nucl. Phys. B442, 593 (1995).

[15] E. Witten, Nuc. Phys. B322, 629 (1989).

[16] R. Gambini, J. Griego, J. Pullin, Phys. Lett. B (to appear).

[17] S. Martin, Nuc. Phys. B338, 244 (1990).

[18] L. Kauffman, S. Lins, "Temperley-Lieb recoupling theory and invariants of 3-Manifolds", Annals of Mathematics Studies, Princeton University Press, Princeton (1994).

[19] M. Alvarez, J. M. F. Labastida, Nucl. Phys. B433 (1995); Erratum B441, 403 (1995); B488, 677 (1997); q-alg/9604010.

[20] See for instance, C. Itzykson, J. Zuber, "Quantum field theory", Mc Graw-Hill, New York (1980).

[21] D. Varshalovich, A. Moskalev, V. Khersonskii, "Quantum theory of angular momentum", World Scientific, Singapore, (1988).

[22] A. Ashtekar, J. Lewandowski, J. Math. Phys. 5, 2170 (1995).

[23] R. Gambini, J. Pullin, "Variational derivations of exact skein relations for Chern-Simons theories", Commun. Math. Phys. 185, 621 (1997).

[24] L. Kauffman, in "Knots and quantum gravity", J. Baez editor, Oxford University Press, Oxford (1993).

[25] D. Altschuler, L. Freidel, Commun. Math. Phys. 170, 41 (1995). 